\title{
Is unexplained elevated maternal serum alpha-fetoprotein still important predictor for adverse pregnancy outcome?
}

\author{
Derya Başbuğ ${ }^{1}$, Alper Başbuğ ${ }^{2}$, Cavidan Gülerman ${ }^{3}$ \\ ${ }^{1}$ Private Clinic, Duzce, Turkey \\ ${ }^{2}$ Düzce University, Faculty of Medicine, Duzce, Turkey \\ ${ }^{3}$ Zekai Tahir Burak Woman's Healt Research and Education Hospital, Ankara, Turkey
}

\begin{abstract}
Objectives: The purpose of this study was to determined the predictive value of maternal serum alpha-fetoprotein (MSAFP) as a marker for adverse pregnancy outcomes.

Material and methods: This study was carried out at Dr. Zekai Tahir Burak Women's Health Education and Research Hospital between 2009 and 2010. This study included a total of 1,177 pregnant women, including 170 in the study group and 1,007 in the control group. Pregnancy outcomes and characteristics were analyzed with regard to the MSAFP value.

Results: Gestational week, birth weight and APGAR scores were significantly lower in the elevated MSAFP group $(p<0.001)$. Adverse pregnancy outcomes, such as preterm delivery, preterm premature rupture of membranes (PPROM), oligohydramnios and intrauterine growth restriction (IUGR) rates were increased in the elevated MSAFP group.

Conclusions: Although ultrasound outweighs as a screening method for neural tube defects and non-invasive prenatal testing outweighs for aneuploidy screening MSAFP level in the second trimester is still an important predictor for poor maternal/fetal outcomes.
\end{abstract}

Key words: alpha-fetoprotein, adverse pregnancy outcome, prenatal tests

Ginekologia Polska 2017; 88, 6: 325-330

\section{INTRODUCTION}

Alpha-fetoprotein (AFP) is a glycoprotein produced by the fetal yolk sac, liver and gastrointestinal system in early pregnancy [1]. Traditionally, the measurement of MSAFP has been the primary screening test used to identify pregnancies at an increased risk of open neural tube defects (NTDs) in women without risk factors for NTDs. The American Congress of Obstetricians and Gynecologists (ACOG) recommends AFP screening for all pregnant women in the second trimester [2]. This is one part of the scanning of multiple serum markers, usually recommended to be done throughout 15 to 20 weeks of pregnancy. In most laboratories, using 2.0-2.5 MoM as the upper limit of normal maternal serum AFP level, with a $5 \%$ false-positivity rate, the detection rate for anencephaly and spina bifida is $90 \%$ and $80 \%$, respectively [3].
Shortly after the introduction of maternal serum screening for the diagnosis of fetal abnormalities, adverse pregnancy outcomes began to be reported after the detection of unexplained isolated elevation of MSAFP [3-5].

When increased maternal AFP levels are found in pregnant women with a correct gestational age, normal fetus structure and normal amniotic fluid AFP levels, the general biological explanation is usually that some damage has occurred at the maternal-fetal interface, which results in elevated levels of AFP in maternal circulation. In a number of studies, using MSAFP cut-off values of 2.0 to $3.0 \mathrm{MoM}$, low birth weight, intrauterine growth retardation (IUGR), premature delivery, placental abruption, intrauterine fetal death (IUFD), preeclampsia and increased risk of perinatal death were found in pregnant women with AFP levels 
above this value [6-7]. Preeclampsia still remains significant cause of maternal and perinatal death, for this reason many researchers focused on the prediction of preeclampsia in the early stages of the pregnancy and they suggested that elevated MSAFP level might be a good predictor for early detection of preeclampsia [8].

In recent years, although ultrasound outweighs as a screening method for neural tube defects, first trimester combined test and non-invasive prenatal testing outweighs for aneuploidy screening. MSAFP level in the second trimester is still an important indicator of pregnancy complications as it is easily accessible and cheap [9, 10].

In this study, we aimed to evaluate the association between unexplained MSAFP elevation in the second trimester with pregnancy complications such as preterm labor, preterm premature rupture of membranes, preeclampsia, IUGR, oligohydramnios, IUFD and placental pathology.

\section{MATERIAL AND METHODS}

This study was carried out at Dr. Zekai Tahir Burak Women's Health Education and Research Hospital, Antenatal outpatient clinic between 2009 and 2010. The study protocol was approved by the local Ethics Committee. The study was conducted in accordance with the principles of the World Medical Association Declaration of Helsinki. The study included primigravida or multiparous pregnant women who were admitted in the second trimester with spontaneous or in vitro fertilization (IVF) singleton pregnancies who underwent triple screening tests.

Using our hospital's central immunoassay laboratory computer records, the total number of patients who underwent triple blood testing in the second trimester was 11,654 . Of these cases, the study group was composed of 243 patients in the second trimester (16-20 weeks of gestation) without chromosomal disorders with an adjusted MSAFP of $\geq 2.0$ MoM for the triple testing which could not be explained by multiple pregnancy, fetal abnormality or fetomaternal hemorrhage. In the next step, ultrasound results of these pregnant women detected five twin pregnancies, 13 fetal anomalies and three hematomas. As a result, 21 pregnant women were excluded from the study group. Another 52 women who were admitted to our hospital, but were lost to follow-up were also excluded. Thus, in total, 170 pregnant women with an unexplained elevation of MSAFP ( $\geq 2.0 \mathrm{MoM}$ ) detected during triple testing years were included in the study group.

Pregnant women without fetal anomalies or chromosomal disorders whose corrected MSAFP, human chorionic gonadotropin (hCG) and unconjugated estriol (uE3) MoM values for the triple testing were within normal range during the same time period were selected for the control group in our study. Those having MSAFP values of $0.75-2.0$ MoM, hCG of 0.5-2.0 MoM and uE3 of above 0.75 MoM were accepted as normal. From 11,654 pregnant women, 6,760 women were with normal corrected MoM values for all parameters of the triple screening test (MSAFP, hCG and $\mathrm{uE}_{3}$ ). At least seven follow-up visits were performed for each case and with a sampling rate of nearly $15 \%$, a total of 1,014 women were chosen. Of these 1,014 randomly selected cases, six fetal anomalies and one twin pregnancy were detected and were excluded from the control group. The total number of the control subjects was 1007 . This study finally included a total of 1,177 pregnant women, including 170 in the study group and 1,007 in the control group.

The gestational age was estimated according to the number of days from the date of the last menstrual period or by ultrasound. In the initial stage, the pregnant women underwent ultrasound examination and the fetal biparietal diameter (BPD), femur length (FL) and abdominal circumference $(A C)$ were measured. The results were recorded in the triple test form files and venous blood samples were drawn from the pregnant women on the same day. Following ultracentrifugation, the AFP, hCG and uE3 levels in the non-hemolyzed and non-lipemic serum samples were measured. For the measurement of serum biochemical markers, the solid-phase, enzyme-labeled chemiluminescent immunometric assay technique was employed using the IMMULITE 2000 kits (Siemens Healthcare, Erlangen, Germany) (sensitivity: for AFP $0.2 \mathrm{lU} / \mathrm{mL}$, for hCG $0.4 \mathrm{mlU} / \mathrm{mL}$ and for uE3 $0.1 \mathrm{ng} / \mathrm{mL}$ ). Using the PRISCA Prenatal Risk Calculation Software 4.0 program (Siemens Healthcare, Erlangen Germany), the corrected MoM values were calculated according to the gestational age, maternal weight, diabetes status, race and smoking habits, and a statistical risk assessment was made for trisomy 21, trisomy 18 and NTD. In the next step, all pregnant women were examined by ultrasound to evaluate fetal abnormalities, chromosomal abnormalities and the presence of fetomaternal hemorrhage.

In this study, the upper limit of MSAFP MoM, adjusted according to the gestational age, maternal weight, smoking habits and diabetes status, was accepted as $2.0 \mathrm{MoM}$ [11].

Data including age, gravida, parity, the number of live births, abortus and curettage, weight, smoking and drug history, gestational diabetes mellitus (GDM) status, IVF, amniocentesis, threatened abortion and threatened preterm labor of 1,177 pregnant women were retrospectively collected from the case files or via phone calls with the patients. Gestational age, mode of delivery, sex of infant, one and five-min Appearance, Pulse, Grimace, Activity, Respiration (APGAR) scores and newborn intensive care needs were assessed.

With the exception of cases of IUFD, those infants having one and five-min APGAR scores above 7 were accepted as normal, while those with APGAR scores below 7 were considered to be abnormal. 
Pregnancy complications involved preterm delivery (delivery before 37 weeks of gestation), PPROM (membrane rupture before 37 weeks of gestation and onset of the uterine concractions, oligohydroamnios (amniotic fluid index $\leq 5$ ), preeclampsia (new onset of hypertension and either proteinuria or end-organ dysfunction after 20 weeks of gestation in a previously normotensive woman) and IUGR (estimated fetal weight $<10^{\text {th }}$ percentile).

A normal pregnancy outcome was defined as birth of a baby after the $37^{\text {th }}$ week, with birth weight being in the 10 percentile according to the gestational age, following a pregnancy free from complications.

\section{Statistical analysis}

The descriptive statistics for continuous variables were expressed in mean \pm standard deviation or median (minimum-maximum), while nominal variables were expressed in the number and percentage (\%). The significance of the difference between the mean values of the groups was evaluated using the Student's t-test, while the significance of the difference in the median values was evaluated using the Mann-Whitney $U$ test. Whether or not AFP had a statistical significant effect on the occurrence of pregnancy complications was assessed using the Pearson's chi-square or Fisher's exact outcome chi-square tests. For the emergence of any pregnancy complication related to AFP, the odds ratio (95\% confidence interval $[\mathrm{CI}]$ ) was calculated. For the prediction of IUFD related to AFP, sensitivity, specificity and positive predictive values (PPVs) and negative predictive values (NPVs) were also calculated. A $p$ value of $<0.05$ was considered statistically significant. Statistical analysis was performed using SPSS for Windows version 17 software (SPSS Inc., Chicago, IL, USA).

\section{RESULTS}

In the study group, the unexplained elevated MSAFP levels ( $\geq 2 \mathrm{MoM}$ ) in the 170 pregnant women in the second trimester triple test were examined. In the control group, 1,007 pregnant women were evaluated and all parameters of the second trimester triple test were found to be normal and without fetal or chromosomal anomalies.

The demographic characteristics of the unexplained elevated MSAFP and control groups are shown in Table 1. The demographic characteristics of the patients were not significantly different statistically.

The distribution of neonatal outcomes and obstetric characteristics of the unexplained elevated MSAFP and control groups can be seen in Table 2.

The mean gestational age at birth was $35.1 \pm 6.5$ weeks in the unexplained elevated MSAFP group and $38.7 \pm 2.3$ weeks in the control group. The gestational age of the study group was significantly lower, compared to the control group $(p<0.001)$.
Table 1. Demographic characteristics of the elevated MSAFP and control groups

\begin{tabular}{|l|c|c|c|}
\hline Variables & $\begin{array}{c}\text { MSAFP } \geq \mathbf{2} \\
\text { MoM } \\
(\boldsymbol{n}=170)\end{array}$ & $\begin{array}{c}\text { MSAFP }<2 \\
\text { MoM } \\
(\boldsymbol{n}=1007)\end{array}$ & $\boldsymbol{p}$ value \\
\hline Age & $27.1 \pm 5.8$ & $26.7 \pm 5.2$ & 0.435 \\
\hline Gravida & $2(1-13)$ & $2(1-9)$ & 0.452 \\
\hline Parity & $1(0-5)$ & $1(0-4)$ & 0.351 \\
\hline Live births & $1(0-10)$ & $1(0-4)$ & 0.556 \\
\hline Abortus & $0(0-8)$ & $0(0-8)$ & 0.926 \\
\hline D \&C & $0(0-7)$ & $0(0-6)$ & 0.196 \\
\hline Smoking history & $14(8.2 \%)$ & $77(7.6 \%)$ & 0.790 \\
\hline Maternal weight $[\mathrm{kg}]$ & $64.5 \pm 11.4$ & $64.5 \pm 11.3$ & 0.959 \\
\hline IVF & $3(1.8 \%)$ & $16(1.6 \%)$ & 0.747 \\
\hline DM & $9(5.3 \%)$ & $31(3.1 \%)$ & 0.140 \\
\hline
\end{tabular}

D \& C - dilatation and curettage; DM - diabetes mellitus; IVF — in vitro fertilization

Table 2. Elevated MSAFP and control group obstetric characteristics and neonatal outcomes

\begin{tabular}{|c|c|c|c|}
\hline Variables & $\begin{array}{c}\text { MSAFP } \geq 2 \\
\text { MoM } \\
(n=170)\end{array}$ & $\begin{array}{c}\text { MSAFP }<2 \\
\text { MoM } \\
(n=1007)\end{array}$ & $p$ value \\
\hline Weeks at birth & $35.1 \pm 6.5$ & $38.7 \pm 2.3$ & $<0.001$ \\
\hline Mode of delivery & & & 0.096 \\
\hline Normal & $\begin{array}{c}60.0 \% \\
(n=102)\end{array}$ & $\begin{array}{c}53.1 \% \\
(n=535)\end{array}$ & \\
\hline $\mathrm{C} / \mathrm{S}$ & $\begin{array}{c}40.0 \% \\
(n=68)\end{array}$ & $\begin{array}{c}46.9 \% \\
(n=472)\end{array}$ & \\
\hline Birth weight [g] & $\begin{array}{c}2930 \\
(560-4490)\end{array}$ & $\begin{array}{c}3300 \\
(288-5040)\end{array}$ & $<0.001$ \\
\hline Sex of child & & & 0.588 \\
\hline Female & $\begin{array}{l}47.1 \% \\
(n=80)\end{array}$ & $\begin{array}{c}49.3 \% \\
(n=496)\end{array}$ & \\
\hline Male & $\begin{array}{l}52.9 \% \\
(n=90)\end{array}$ & $\begin{array}{c}50.7 \% \\
(n=510)\end{array}$ & \\
\hline NICU required & $\begin{array}{l}21.8 \% \\
(n=37)\end{array}$ & $\begin{array}{c}6.3 \% \\
(n=63)\end{array}$ & $<0.001$ \\
\hline APGAR & & & $<0.001$ \\
\hline Normal & $\begin{array}{c}75.9 \% \\
(n=129)\end{array}$ & $\begin{array}{c}98.7 \% \\
(n=993)\end{array}$ & \\
\hline Low & $\begin{array}{c}6.5 \% \\
(n=11)\end{array}$ & $\begin{array}{l}0.6 \% \\
(n=6)\end{array}$ & \\
\hline
\end{tabular}

APGAR - Appearance, Pulse, Grimace, Activity, Respiration; $\mathrm{C} / \mathrm{S}$ - cesarean section; ICU — intensive care unit

The mean birth weight was 2,930 $\mathrm{g}$ in the unexplained elevated MSAFP group and 3,300 $\mathrm{g}$ in the control group. The birth weight in the study group was significantly lower than in the control group $(p<0.001)$.

The offsprings of 37 women (21.8\%) in the elevated MSAFP group, compared to those of $63(6.3 \%)$ in the control 
Table 3. High MSAFP values $(\geq 2.0 \mathrm{MoM})$ in relation to pregnancy complications

\begin{tabular}{|c|c|c|c|c|}
\hline Pregnancy complications & Present & Absent & $p$ values & Odds ratio $(95 \% \mathrm{CI})$ \\
\hline \multicolumn{5}{|l|}{ Preterm delivery } \\
\hline $\mathrm{AFP} \geq 2 \mathrm{MoM}$ & $29(47.1 \%)$ & $141(12.7 \%)$ & 0.001 & $5.547(3.298-9.331)$ \\
\hline AFP $<2$ MoM & $36(52.9 \%)$ & $971(87.3 \%)$ & - & 1.000 \\
\hline \multicolumn{5}{|l|}{ PPROM } \\
\hline $\mathrm{AFP} \geq 2 \mathrm{MoM}$ & $13(41.9 \%)$ & $157(13.7 \%)$ & 0.001 & $4.550(2.186-9.469)$ \\
\hline AFP $<2$ MoM & $18(58.1 \%)$ & $989(86.3 \%)$ & - & 1.000 \\
\hline \multicolumn{5}{|l|}{ Oligohydramnios } \\
\hline $\mathrm{AFP} \geq 2 \mathrm{MoM}$ & $21(31.3 \%)$ & 49 (13.4\%) & 0.001 & $2.944(1.709-5.074)$ \\
\hline AFP $<2 \mathrm{MoM}$ & $46(68.7 \%)$ & $961(86.6 \%)$ & - & 1.000 \\
\hline \multicolumn{5}{|l|}{ Preeclampsia } \\
\hline $\mathrm{AFP} \geq 2 \mathrm{MoM}$ & $5(17.9 \%)$ & $165(14.4 \%)$ & 0.586 & $1.296(0.486-3.458)$ \\
\hline AFP $<2 \mathrm{MoM}$ & $23(82.1 \%)$ & $984(85.6 \%)$ & - & 1.000 \\
\hline \multicolumn{5}{|l|}{ IUGR } \\
\hline $\mathrm{AFP} \geq 2 \mathrm{MoM}$ & $24(40.7 \%)$ & $146(13.1 \%)$ & 0.001 & $4.565(2.640-7.895)$ \\
\hline AFP $<2$ MoM & 35 (59.3\%) & $972(86.9 \%)$ & - & 1.000 \\
\hline
\end{tabular}

IUGR — intrauterine growth restriction; PPROM — preterm premature rupture of membranes

group, were referred to the intensive care unit. The study group statistically significantly increased the need for the $\operatorname{NICU}(p<0.001)$.

In the unexplained elevated MSAFP group, 129 infants had normal APGAR scores, while 11 (6.5\%) had low APGAR scores and IUFD occurred in 30 (17.6\%). In the control group, these rates were $993(98.7 \%)$ had normal APGAR scores, 6 had low APGAR scores ( $0.6 \%$ ) and 7 IUFD occurred ( $0.7 \%$ ). The offsprings with low APGAR scores and the IUFD cases in the study group were significantly higher, compared to the control group $(p<0.001)$.

The pregnancies of 29 women in the unexplained elevated MSAFP group and 36 in the control group were complicated by preterm births. The high risk of occurrence of preterm delivery in the study group was statistically significant compared to the control group, with an odds ratio (OR) of 5.547 (95\% Cl 3.298-9.331; $p<0.001)$. In the unexplained elevated MSAFP group, 13 out of 36 pregnant women had preterm births caused by preterm premature rupture of membranes (PPROM), while in the control group, 18 out of 29 preterm births were due to PPROM.

Preterm premature rupture of membranes was observed in 13 pregnant women in the unexplained elevated MSAFP group and 18 in the control group, indicating a significantly higher risk for developing PPROM in the study group with an OR of 4.550 (95\% Cl 2.186-9.469; $p<0.001)$.

Oligohydramnios was detected in 31 pregnant women in the unexplained elevated MSAFP group and 58 in the control group. Upon detection in these cases, the accompanying intrauterine growth restriction (IUGR) was evaluated in the 10 out of these 31 women in the unexplained elevated MSAFP group and in the 12 out of 58 in the control group. Isolated oligohydramnios was present in 21 pregnant women in the unexplained elevated MSAFP group and in 46 women in the control group. Compared to the control group, the risk of oligohydramnios was significantly higher in the study group with an OR of 2.944 (95\% Cl 1.709-5.074; $p<0.001)$.

Preeclampsia complications were present in five pregnant women in the unexplained elevated MSAFP group and 23 in the control group. In terms of preeclampsia, there was no statistically significant difference between the groups with an OR of 1.296 (95\% Cl 0.486-3.458; $p=0.586$ ).

The presence of IUGR complications was found in 24 women in the study group and 35 in the control group. Compared to the control group, the risk of IUGR was found to be significantly higher in the study group with an OR of 4.565 (95\% Cl 2.640-7.895; $p<0.001)$.

In addition, 91 women in the unexplained elevated MSAFP group and 138 in the control group experienced any type of pregnancy complication. Compared to the control group, the risk of developing any type of pregnancy complication increased by 7.2-fold in the study group with an OR of 7.254 (95\% Cl 5.107-10.302; $p<0.001)$.

The AFP-related sensitivity, specificity, PPVs and NPVs for prediction of adverse pregnancy outcomes are shown in Table 4. The preterm birth prediction for AFP was found to be $44.6 \%$ for sensitivity, $87.3 \%$ for specificity, $17.1 \%$ for PPV and $96.4 \%$ for NPV. The IUGR prediction for AFP was found to be $40.7 \%$ for sensitivity, $86.9 \%$ for specificity, $14.1 \%$ for PPV and $96.5 \%$ for NPV. The AFP-related IUFD prediction for sensitivity was $67.9 \%$ with $86.9 \%$ specificity, $11.2 \%$ PPV and 99.1\% NPV.The AFP prediction for oligohydramnios sensitiv- 
Table 4. AFP-related sensitivity, specificity and PPVs and NPVs for pregnancy complications

\begin{tabular}{|l|c|c|c|c|}
\hline Variables & Sensitivity & Specificity & PPV & NPV \\
\hline Preterm delivery & $44.6 \%$ & $87.3 \%$ & $17.1 \%$ & $96.4 \%$ \\
\hline IUGR & $40.7 \%$ & $86.9 \%$ & $14.1 \%$ & $96.5 \%$ \\
\hline IUFD & $67.9 \%$ & $86.9 \%$ & $11.2 \%$ & $99.1 \%$ \\
\hline Oligohydramnios & $31.3 \%$ & $86.6 \%$ & $12.4 \%$ & $95.4 \%$ \\
\hline PPROM & $41.9 \%$ & $86.3 \%$ & $7.6 \%$ & $98.2 \%$ \\
\hline Preeclampsia & $17.9 \%$ & $85.6 \%$ & $2.9 \%$ & $97.7 \%$ \\
\hline $\begin{array}{l}\text { Adverse perinatal } \\
\text { outcomes }\end{array}$ & $39.7 \%$ & $91.7 \%$ & $53.5 \%$ & $86.3 \%$ \\
\hline
\end{tabular}

IUFD — intrauterine fetal death; IUGR — intrauterine growth restriction; NPV - negative predictive value; PPROM — preterm premature rupture of membranes; PPV - positive predictive value

ity was found to be $31.3 \%$ with $86.6 \%$ specificity, $12.4 \%$ PPV and $95.4 \%$ NPV. The PPROM prediction for AFP sensitivity was found to be $41.9 \%$ with $86.3 \%$ specificity, $7.6 \%$ PPV and 98.2\% NPV. In the prediction of preeclampsia, AFP sensitivity was found to be $17.9 \%$ with $85.6 \%$ specificity, $2.9 \%$ PPV and $97.7 \%$ NPV. In the prediction of any pregnancy complications, AFP sensitivity was $39.7 \%$ with $91.7 \%$ specificity, $53.5 \%$ PPV and $86.3 \%$ NPV (Table 4).

\section{DISCUSSION}

The maternal serum alpha-fetoprotein (MSAFP) levels, other than for NTD screening, with $\beta$-hCG and uE3 (trisomy) screening tests in the second trimester are included in the antenatal care protocol [7]. Several studies have shown that MSAFP levels of $\geq 2$ MoM in the second trimester, except from cases of multiple gestation or fetal anomalies, are associated with an increased risk of preeclampsia, IUGR, preterm delivery, placental abruption and fetal loss [12, 13]. The result of this study showed that unexplained maternal serum AFP levels in the second trimester of pregnancy is related with maternal, fetal and neonatal complications.

To determine the optimal AFP cut-off value, in their meta-analysis, Yuan et al. [14] carried out a systematic study of the relationship between elevated second trimester MSAFP in the general population and preterm delivery. The study examined a total of 207,135 women in 24 studies published between the years 1991 and 2007 including 14 population-based studies and 10 comparative case-control studies. Fourteen of the studies used 2.0 MoM as the elevated AFP level, while eight studies used 2.5 MoM as the cut-off value. In our study evaluating the association between unexplained MSAFP elevation and pregnancy complications, we used the cut-off value for unexplained MSAFP as $\geq 2$ MoM, which significantly increased the risk of pregnancy complications such as preterm delivery, PPROM, IUGR and oligohydramnios in the pregnant women.
Morris et al. performed a systematic meta-analysis of cohort studies evaluating second trimester markers and preeclampsia. There was significant variation among studies in the threshold used to identify patients at high-risk as well as significant variation in screening performance. The most effective thresholds were 2.0 multiples of the median (MoM) for alpha-fetoprotein (AFP) resulting in a positive likelihood ratio (LR) of 2.36 and a negative likelihood ratio of 0.96 [15]. On the other hand Davidson, Kang and Wald did not find any significant increase in MSAFP levels in preeclamptic women $[8,16,17]$. We found no significant difference in the rate of preeclampsia between the groups. The relatively small sample size of the preeclampsia in the elevated MSAFP group is a limitation of this study.

Katz et al. [18] in the compilation of their publications, found that after excluding anomalies evaluated by ultrasound and/or amniocentesis, elevated MSAFP, preterm delivery and IUGR were associated with a 2 to 4 -fold increase in the risk of giving birth to a low-birth weight baby. Meanwhile, unexplained elevated AFP levels (2.0-3.0 MoM) were associated with a 10-fold increase in placental abruption and perinatal mortality. The results obtained from the scans of 225,000 pregnant women showed that 20 to $38 \%$ of the women with unexplained MSAFP elevation had poor pregnancy outcomes. Similarly, our study results are consistent with the previous findings.

In a prospective, multi-center, cohort study Smith etal.[19] evaluated 8,483 pregnant women attending a prenatal program in Scotland over a two-year period. The patients were examined for the relationship between MSAFP levels and adverse perinatal outcome. In women with a high MSAFP level, a statistically significant increased risk of preterm birth with an OR of 2.1 and stillbirth with an OR of 4.4 was found. These values are also consistent with our findings.

In another study, Anfuso et al. [20] conducted a retrospective cohort study of pregnant women having unexplained high MSAFP levels ( $\geq 2.5 \mathrm{MoM}$ ) due to any visible cause and compared them to women with normal MSAFP levels. They were found to have a 5.8-fold increased risk of preterm birth, a 15.2-fold increased risk of PRROM, a 2.6-fold increased risk of preeclampsia/HELLP syndrome, a 5.9-fold increased risk of IUGR and a significantly increased risk of IUFD. Similarly, in our study, we identified the risks of preterm delivery, PPROM, IUGR and IUFD to be significantly increased. However, unlike Anfuso et al. we were unable to find any statistically significant difference in terms of preeclampsia between the control and the study groups.

\section{CONCLUSIONS}

In conclusion, our study results showed that unexplained second trimester MSAFP elevation was significantly associated with poor maternal/fetal outcomes. In patients 
where the fetus is structurally normal, elevated MSAFP indicates a defect in the placentation.

Although unexplained high MSAFP values are associated with an increased risk of pregnancy complications, there is no consensus on the need for the observation of these pregnant women or on the methods to be implemented for monitoring these patients. Due to the low sensitivity of MSAFP and its PPV, its use alone as a screening test to predict pregnancy complications can be considered appropriate. Therefore, these pregnant women should be under close clinical follow-up and auxiliary testing should be carried out to improve maternal/fetal outcome and, eventually, to reduce perinatal mortality and morbidity. Furthermore, AFP with other serum markers, particularly hCG, in combination with uterine artery Doppler analysis can increase the recognition of pregnancy complications in women. In addition, detailed pathological examination of the placenta following delivery can provide further information about the cause of MSAFP elevation.

\section{Conflict of interest}

The authors report no conflict of interest.

\section{REFERENCES}

1. Cunningham FG, Lenevo KJ, Bloom SL. Williams Obstetrics. Twenty-Third Edition. McGraw-Hill Companies, New York 2010: 288-289.

2. Milunsky A, Canick JA. Maternal serum screening for neural tube and other defects. In: Milunsky A, Canick JA. ed. Genetic disorders and the fetus. Diagnosis, prevention, and treatment, 5th ed. The Johns Hopkins University Press, Baltimore and London 2004: 719.

3. Robinson L, Grau P, Crandall BF. Pregnancy outcomes after increasing maternal serum alpha-fetoprotein levels. Obstet Gynecol. 1989; 74(1): 17-20, indexed in Pubmed: 2471946.

4. Crandall BF, Robinson L, Grau P. Risks associated with an elevated maternal serum alpha-fetoprotein level. Am J Obstet Gynecol. 1991; 165(3): 581-586, indexed in Pubmed: 1716419.

5. Haddow JE, Kloza EM, Smith DE, et al. Data from an alpha-fetoprotein pilot screening program in Maine. Obstet Gynecol. 1983; 62(5): 556-560, indexed in Pubmed: 6194484.

6. Milunsky A. Maternal serum screening for neural tube and other defects. In: Milunsky A. ed. Genetic disorders and the fetus. Diagnosis, prevention, and treatment, 3rd ed. The Johns Hopkins University Press, Baltimore 1992: 507-656.
7. Wapner RJ, Jenkins TM, Khalek N. Prenatal Diagnosis of Congenital Disorders. In: Creasy RK, Resnik R, lams JD, Lockwood CJ, Moore T. ed. Creasy and Resnik's Maternal-Fetal Medicine: Principles and Practice, 6th ed. 2009: 221-226.

8. Davidson E, Riley S, Roberts $S$, et al. Maternal serum activin, inhibin, human chorionic gonadotrophin and alpha-fetoprotein as second trimester predictors of pre-eclampsia. BJOG: An International Journal of Obstetrics and Gynaecology. 2003; 110(1):46-52, doi: 10.1046/j.1471-0528.2003.02037.x.

9. Bartkute K, Balsyte D, Wisser J, et al. Pregnancy outcomes regarding maternal serum AFP value in second trimester screening. J Perinat Med. 2016 [Epub ahead of print], doi: 10.1515/jpm-2016-0101, indexed in Pubmed: 27771626.

10. Tancrède $S$, Bujold $E$, Giguère $Y$, et al. Mid-trimester maternal serum AFP and hCG as markers of preterm and term adverse pregnancy outcomes. J Obstet Gynaecol Can. 2015; 37(2): 111-116, indexed in Pubmed: 25767942.

11. Androutsopoulos G, Gkogkos P, Papadopoulos V, et al. Mid-trimester maternal serum AFP levels in predicting adverse pregnancy outcome. Clin Exp Obstet Gynecol. 2009; 36(4): 237-240, indexed in Pubmed: 20101856.

12. Wilkins-Haug L. Unexplained elevated maternal serum alpha-fetoprotein: what is the appropriate follow-up? Curr Opin Obstet Gynecol. 1998; 10(6): 469-474, indexed in Pubmed: 9866015.

13. Waller DK, Lustig LS, Cunningham GC, et al. The association between maternal serum alpha-fetoprotein and preterm birth, small for gestational age infants, preeclampsia, and placental complications. Obstet Gynecol. 1996; 88(5): 816-822, indexed in Pubmed: 8885920.

14. Yuan W, Chen L, Bernal AL. Is elevated maternal serum alpha-fetoprotein in the second trimester of pregnancy associated with increased preterm birth risk? A systematic review and meta-analysis. Eur J Obstet Gynecol Reprod Biol. 2009; 145(1): 57-64, doi: 10.1016/j.ejogrb.2009.04.017, indexed in Pubmed: 19457604

15. Morris RK, Cnossen JS, Langejans M, et al. Serum screening with Down's syndrome markers to predict pre-eclampsia and small for gestational age: systematic review and meta-analysis. BMC Pregnancy Childbirth. 2008; 8: 33, doi: 10.1186/1471-2393-8-33, indexed in Pubmed: 18680570.

16. Kang JH, Farina A, Park JiH, et al. Down syndrome biochemical markers and screening for preeclampsia at first and second trimester: correlation with the week of onset and the severity. Prenat Diagn. 2008; 28(8): 704-709, doi: 10.1002/pd.1997, indexed in Pubmed: 18655226.

17. Wald NJ, Morris JK. Multiple marker second trimester serum screening for pre-eclampsia. J Med Screen. 2001; 8(2):65-68, doi: 10.1136/jms.8.2.65, indexed in Pubmed: 11480445

18. Katz VL, Chescheir NC, Cefalo RC. Unexplained elevations of maternal serum alpha-fetoprotein. Obstet Gynecol Surv. 1990; 45(11): 719-726, indexed in Pubmed: 1700347.

19. Smith GCS, Shah I, Crossley JA, et al. Pregnancy-associated plasma protein $A$ and alpha-fetoprotein and prediction of adverse perinatal outcome. Obstet Gynecol. 2006; 107(1): 161-166, doi: 10.1097/01. AOG.0000191302.79560.d8, indexed in Pubmed: 16394054.

20. Anfuso $S$, Soncini $E$, Bonelli $P$, et al. Second-trimester maternal serum alpha-fetoprotein elevation and its association with adverse maternal/fetal outcome: ten years experience. Acta Biomed. 2007; 78(3): 214-219, indexed in Pubmed: 18330082. 